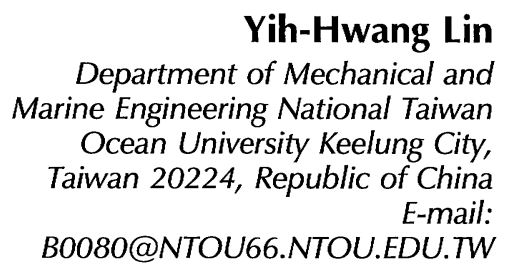

Yih-Hwang Lin Ocean University Keelung City,

B0080@NTOU66.NTOU.EDU.TW

\section{Optimal Vibration Suppression in Modal Space for Flexible Beams Subjected to Moving Loads}

\begin{abstract}
Optimal independent modal space control for vibration suppression of beam structures traversed by a moving concentrated force was examined. Two control methodologies, optimal linear quadratic tracking and an optimal linear quadratic regulator, were utilized, with the former approach taking into account the disturbance due to the moving load and the latter one simply ignoring that disturbance. One single actuator placed at the beam center was found to be sufficient to suppress excessive vibration of a beam traversed by the moving load with a reasonable amount of control input. This study shows that excessive vibration of the beam structure induced by the moving load can be more effectively suppressed using the tracking control approach than using the regulator design, even with $\pm 50 \%$ variation of the moving load magnitude or speed from that designed for the tracking control system. (c) 1997 John Wiley \& Sons, Inc.
\end{abstract}

\section{INTRODUCTION}

The dynamic analysis of structures subjected to moving loads has attracted much attention from engineers and scientists since the 19th century (Stokes, 1883). A dedicated monograph was written by Fryba on the subject of moving load problems (1972). When a simply supported beam was subjected to a moving force traveling at constant velocity, the maximum dynamic beam displacement was found to be about $50 \%$ greater than that of the static case when the concentrated force was moving at a velocity corresponding to the first resonant beam mode (Timoshenko, 1955). It was later found that the maximum dynamic response can be as high as 1.743 times that of static deflection when the traveling time to cross the beam span is 0.81 times the fundamental period of the beam (Warburton, 1976). Besides the original concerns for dynamic analysis of railway bridges and highway structures subjected to moving vehicle loads, the case of a rotating beam subjected to deflection-dependent moving forces, pertinent for the study of lathe machining operation, was studied (Katz et al., 1987). To handle more complex situations, finite element methods were applied by Filho (1978), Hino et al. (1984, 1985), and Yoshimura et al. (1986). A general treatment for various moving load problems and application to a high speed industrial precision drilling machine were reported by Lin and Trethewey (1990) and Lin et al. (1990).

In spite of the intense research activities on the dynamic analysis of structures subjected to moving

Received May 24, 1995; Accepted July 1, 1996. 
loads, research effort on the effective suppression of excessive vibration induced by the moving loads is quite limited and an in-depth study to ensure structural safety and operation accuracy is required. Lin and Cho (1993) examined the performance of a passive technique for reducing structural vibration for systems excited by moving loads. A coupled mode control formulation on the moving load problem was reported by $\mathrm{Su}$ and Tadjbakhsh (1991) with control forces determined from the past information only. Another straightforward coupled mode control scheme using the finite element method for a fixed-fixed beam system traversed by a moving load was developed by Lin and Trethewey (1993). The formulation resulted in the requirement of solving a set of 78 first-order simultaneous nonlinear differential equations for the elements in the Riccati matrix and another set of 12 first-order differential equations with time-dependent coefficients while only four finite elements were used in the model. The purpose of this study is to alleviate such demanding computational requirements by applying the independent modal space control technique (Meirovitch and Baruh, 1982) and to assess the obtainable level of vibration reduction with respect to the level of the control actuation. With the use of this formulation, only a set of three firstorder simultaneous nonlinear differential equations for the elements in the Riccati matrix and another set of two first-order differential equations with time-dependent coefficients need to be solved for each mode controlled. Robustness of this modal control technique for systems with uncertain parameters has been demonstrated (Meirovitch and Baruh, 1983; Baruh and Silverberg, 1985). In this work, optimal tracking control formulation combined with the independent modal space control technique are developed to take into account the disturbance caused by the moving load. The regulator control approach is also analyzed for comparison to assess the effectiveness of the tracking control design.

In the following sections, first the equation of motion for a beam system subjected to a moving force traveling at constant velocity along with the control input is given. Next, the governing equation is transformed to a modal coordinate to enable independent control in modal space. Optimal tracking control is applied to obtain the feedback gains by minimizing a cost function defined as the weighted sum of kinetic energy, potential energy, and the control effort. Finally, the numerical implementation considerations and the performance of the control system designed are discussed.

\section{MODEL DEVELOPMENT}

Figure 1 depicts the moving load problem being considered. A simply supported Bernoulli-Euler beam is subjected to a moving force traveling at a constant velocity. The equation of motion can be described as

$$
E I \frac{\partial^{4} y}{\partial x^{4}}+\gamma \frac{\partial^{2} y}{\partial t^{2}}=F_{0} \delta(x-v t)+u(x, t),
$$

where $E I$ is the bending rigidity, $\gamma$ the mass density per unit length of the beam, $F_{0}$ magnitude of the moving force, $\delta$ the Dirac delta function, and $u(x, t)$ the control input force to be designed. The moving force can be described by using the Fourier series expansion as

$$
F_{0} \delta(x-v t)=\frac{2 F_{0}}{L} \sum_{n=1}^{\infty} \sin \frac{n \pi v t}{L} \sin \frac{n \pi x}{L} .
$$

At the current state of the art for active structural control, distributed actuation on a real structure is generally not possible and the control action is usually provided by discrete actuators as shown in Fig. 1. For discrete actuation, the control input $u(x, t)$ can be described as

$$
u(x, t)=\sum_{k=1}^{m} u_{k}(t) \delta\left(x-x_{k}\right)
$$

in which $m$ and $x_{k}$ denote the number and position of the actuators, respectively.

The boundary conditions for the simply supported beam considered here are

$$
y(0, t)=y(L, t)=0
$$

and

$$
\left.\frac{\partial^{2} y}{\partial^{2} x}\right|_{x=0}=0 ;\left.\quad \frac{\partial^{2} y}{\partial x^{2}}\right|_{x=L}=0 .
$$

Using the expansion theorem

$$
y(x, t)=\sum_{i=1}^{\infty} \phi_{i}(x) q_{i}(t),
$$




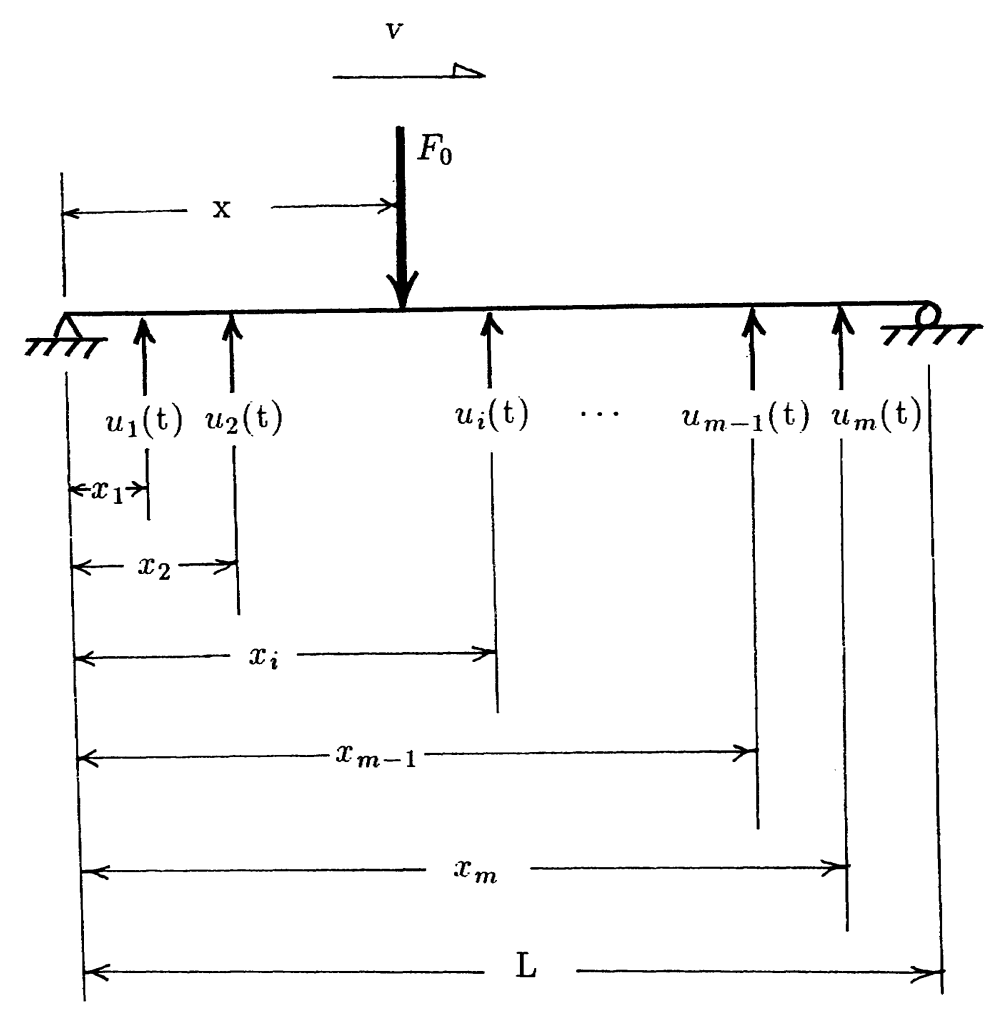

FIGURE 1 A beam traversed by a moving concentrated force.

and knowing that $\phi_{i}(x)=\sin i \pi x / L$ are the mode shape functions for the beam system examined, Eq. (1) can be transformed to a set of an infinite number of ordinary differential equations by substituting Eq. (6) into Eq. (1) and applying the orthogonality of mode shapes to obtain

$$
\ddot{q}_{i}(t)+\omega_{i}^{2} q_{i}(t)=p_{i}(t)+f_{i}(t), \quad i=1,2, \ldots, \infty
$$

where

$$
p_{i}(t)=\frac{2 F_{0}}{\gamma L} \sin \frac{i \pi v t}{L}
$$

and

$$
f_{i}(t)=\frac{2}{\gamma L} \sum_{k=1}^{m} u_{k}(t) \sin \frac{i \pi x_{k}}{L} .
$$

Equation (7) can be completely decoupled if the modal control action $f_{i}(t)$ depends on $\dot{q}_{i}(t)$ and $q_{i}(t)$ only, which is the essence of the independent modal space control technique. In practice, it is not feasible to control the innumerable structural modes, nor is it necessary to do so because the higher modes are more difficult to be excited, and the control of the first few modes will usually be sufficient. To control the first $n$ modes, let

$$
\begin{aligned}
& \mathbf{q}_{\mathbf{c}}(t)=\left\lfloor q_{1}(t) \quad q_{2}(t) \cdot \cdot q_{n}(t)\right\rfloor, \\
& \mathbf{p}(t)=\left\lfloor p_{1}(t) \quad p_{2}(t) \cdot \cdot p_{n}(t)\right\rfloor, \\
& \mathbf{f}(t)=\left\lfloor f_{1}(t) \quad f_{2}(t) \cdots f_{n}(t)\right\rfloor^{T}, \\
& \mathbf{u}(t)=\left\lfloor u_{1}(t) \quad u_{2}(t) \cdot \cdot u_{m}(t)\right\rfloor, \\
& \lambda_{c}=\operatorname{diag}\left(\omega_{1}^{2} \quad \omega_{2}^{2} \cdot \cdots \cdot \omega_{n}^{2}\right) \text {. }
\end{aligned}
$$

Thus, the governing equation of motion in modal space can be written as

$$
\ddot{\mathbf{q}}_{\mathrm{c}}(t)+\lambda_{\mathrm{c}} \mathbf{q}_{\mathrm{c}}(t)=\mathbf{p}(t)+\mathbf{f}(t),
$$

where

$$
\mathbf{f}(t)=\mathbf{B u}(t)
$$

and

$$
\begin{aligned}
\mathbf{B} & =\left[\frac{2}{\lambda L} \sin \frac{i \pi x_{k}}{L}\right], \quad i=1,2, \ldots, n, \\
k & =1,2, \ldots, m .
\end{aligned}
$$


The actual control input $\mathbf{u}(t)$ can then be obtained using

$$
\mathbf{u}(t)=\mathbf{B}^{-1} \mathbf{f}(t) .
$$

Note that to avoid unnecessary inaccuracy due to the use of pseudoinversion, the $\mathbf{B}$ matrix should be square, which implies that the number of actuators is equal to that of the modes being controlled. To facilitate the control system design process, the original governing equation is described using the state equation below:

$$
\begin{aligned}
\dot{\mathbf{q}}_{\mathrm{i}}(t) & =\mathbf{A}_{i} \overline{\mathbf{q}}_{\mathrm{i}}(t)+\mathbf{B}_{\mathrm{i}} f_{i}(t)+\overline{\mathbf{P}}_{\mathrm{i}}(t), \\
i & =1,2, \ldots, n,
\end{aligned}
$$

where

$$
\begin{aligned}
\overline{\mathbf{q}}_{\mathbf{i}}(t) & =\left\lfloor q_{i}(t)\right. \\
v_{i}(t) & =\frac{\dot{q}_{i}(t)}{\omega_{i}}, \\
\mathbf{A}_{\mathbf{i}} & =\left[\begin{array}{cc}
0 & \omega_{i} \\
-\omega_{i} & 0
\end{array}\right], \\
\mathbf{B}_{\mathbf{i}} & =\left\lfloor\begin{array}{ll}
0 & \frac{1}{\omega_{i}}
\end{array}\right]^{T}, \\
\overline{\mathbf{P}}_{\mathbf{i}}(t) & =\left\lfloor\begin{array}{ll}
0 & \frac{p_{i}(t)}{\omega_{i}}
\end{array}\right]^{T} .
\end{aligned}
$$

\section{OPTIMAL CONTROL FORMULATION}

The concept of linear quadratic optimal control in dealing with the tracking problem was utilized to design a control system for the model presented in the previous section. The modal objective function, $J_{i}$, to be minimized for the dynamic system described by Eq. (15) is defined as

$$
J_{i}=\frac{1}{2} \mathbf{z}_{\mathbf{i}}^{T}\left(t_{f}\right) \mathbf{S}_{\mathbf{i}} \mathbf{Z}_{\mathbf{i}}\left(t_{f}\right)+\frac{1}{2} \int_{t_{0}}^{t_{f}}\left(\mathbf{z}_{\mathbf{i}}^{T}(t) \mathbf{F}_{\mathbf{i}} \mathbf{z}_{\mathbf{i}}(t)+e_{i} f_{i}^{2}(t)\right) d t,
$$

in which

$$
\mathbf{z}_{\mathrm{i}}(t)=\eta_{\mathrm{i}}(t)-\overline{\mathbf{q}}_{\mathbf{i}}(t),
$$

where the terminal time, $t_{f}$, is specified; $\mathbf{S}_{\mathbf{f}_{\mathbf{i}}}$ and $\mathbf{F}_{\mathbf{i}}$ are $2 \times 2$ positive semidefinite matrices; $e_{i}$ is a $1 \times 1$ positive definite matrix, or a positive scalar number; and $\eta_{\mathrm{i}}(t)$ denotes the desired trajectory of the beam and is set to zero in this study to minimize the beam vibration. The modal control input, $f_{i}(t)$, is assumed to be unconstrained in this formulation.

The first term on the right side of Eq. (17) is used to guarantee the error, $\mathbf{z}_{\mathbf{i}}(t)$, at the terminal time, $t_{f}$, is small. The term $\mathbf{z}_{\mathbf{i}}^{T}(t) \mathbf{F}_{\mathbf{i}} \mathbf{z}_{\mathbf{i}}(t)$ is used to weight the cost of the beam response during the entire period $t_{0}$ to $t_{f}$, while the term $\mathbf{e}_{i} f_{i}^{2}(t)$ weights the control effort so that a reasonable amount of control energy is used. It is the designer's decision to choose proper weighting matrices, $\mathbf{S}_{\mathbf{f}_{i}}, \mathbf{F}_{\mathbf{i}}$, and $e_{i}$ to keep the error, $\mathbf{z}_{i}(t)$, small without using excessively large or unrealistic control forces. In this study, $\mathbf{F}_{\mathbf{i}}=\omega_{i}^{2} \mathbf{I}$ is used, where $\mathbf{I}$ is the $2 \times 2$ identity matrix, and the weighted beam response cost then becomes the total energy associated with the $i$ th mode to be controlled.

Minimization of the objective function, $J_{i}$, shown in Eq. (17) leads to (Kirk, 1970; Anderson and Moore, 1971)

$$
f_{i}(t)=-\frac{1}{e_{i}} \mathbf{B}_{\mathbf{i}}^{T}\left(\mathbf{S}_{\mathbf{i}}(t) \overline{\mathbf{q}}_{\mathbf{i}}(t)-\xi_{\mathrm{i}}(t)\right),
$$

in which $\mathbf{S}_{\mathrm{i}}(t)$ is the so-called Riccati matrix satisfying the following nonlinear matrix differential equation:

$$
\dot{\mathbf{S}}_{\mathbf{i}}(t)+\mathbf{S}_{\mathbf{i}}(t) \mathbf{A}_{\mathbf{i}}+\mathbf{A}_{\mathbf{i}}^{T} \mathbf{S}_{\mathbf{i}}(t)-\frac{1}{e_{i}} \mathbf{S}_{\mathbf{i}}(t) \mathbf{B}_{\mathbf{i}} \mathbf{B}_{\mathbf{i}}^{T} \mathbf{S}_{\mathbf{i}}(t)+\mathbf{F}_{\mathbf{i}}=0
$$

with terminal conditions

$$
\mathbf{S}_{\mathbf{i}}\left(t_{f}\right)=\mathbf{S}_{\mathbf{f}_{\mathbf{i}}} ;
$$

and $\xi_{\mathrm{i}}(t)$ is an open loop control vector to account for the desired output, $\eta_{\mathrm{i}}(t)$, and the disturbance, $\overline{\mathbf{P}}_{\mathrm{i}}(t)$, due to the moving load, that satisfies the following differential equation with time-dependent coefficients:

$$
\begin{array}{r}
-\dot{\xi}_{\mathrm{i}}(t)+\left(\frac{1}{e_{i}} \mathbf{S}_{\mathbf{i}}(t) \mathbf{B}_{\mathbf{i}} \mathbf{B}_{\mathbf{i}}^{T}-\mathbf{A}_{\mathbf{i}}^{T}\right) \\
\xi_{\mathbf{i}}(t)+\mathbf{S}_{\mathbf{i}}(t) \overline{\mathbf{P}}_{\mathbf{i}}(t)-\mathbf{F}_{\mathbf{i}} \eta_{\mathbf{i}}(t)=0,
\end{array}
$$

with terminal conditions

$$
\xi_{\mathbf{i}}\left(t_{f}\right)=\mathbf{S}_{\mathbf{i}_{\mathrm{i}}} \eta_{\mathbf{i}}\left(t_{f}\right)
$$




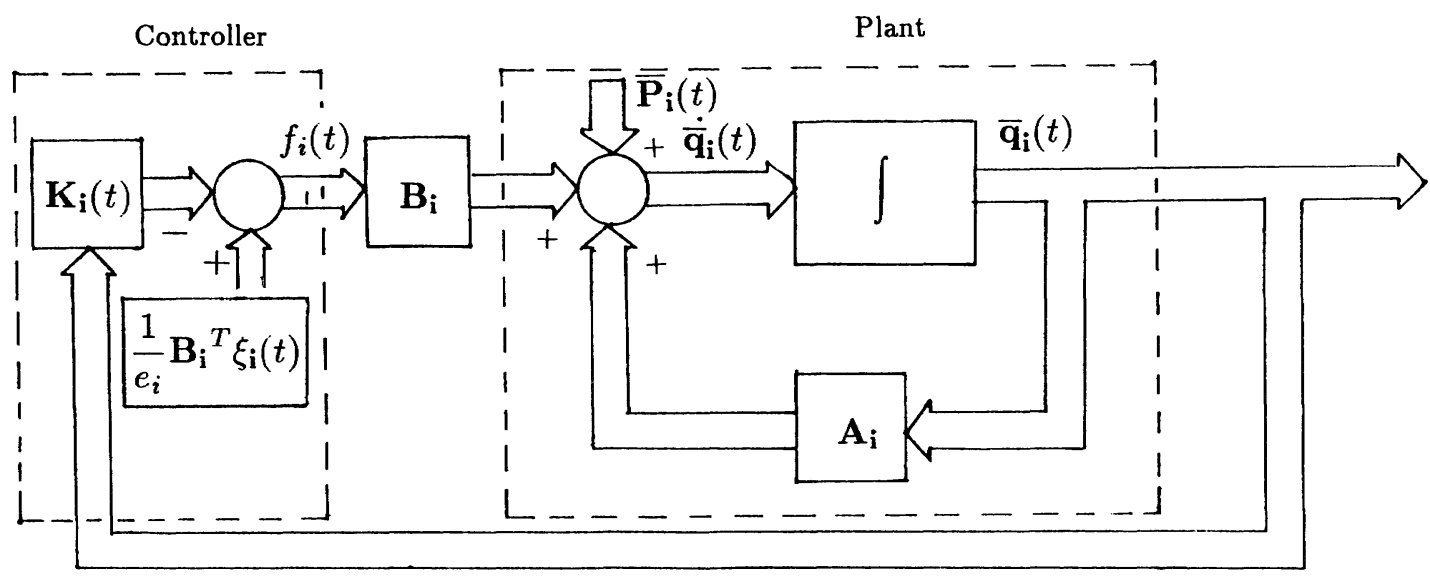

FIGURE 2 Optimal modal feedback controller and plant.

The Riccati matrix, $\mathbf{S}_{\mathrm{i}}(t)$, shown in Eq. (20) is symmetric and thus only three nonlinear differential equations need to be solved using backward integration. Once the Riccati matrix is determined, it can then be used in Eq. (21), which is also integrated backward, to obtain the $\xi_{\mathrm{i}}(t)$ vector. Note that both the Riccati matrix, $\mathbf{S}_{\mathrm{i}}(t)$, and the $\xi_{\mathrm{i}}(t)$ vector can be solved off-line because they are independent of the modal states.

From Eq. (19), the modal control input, $f_{i}(t)$, may also be expressed as

$$
f_{i}(t)=-\mathbf{K}_{\mathbf{i}}(t) \overline{\mathbf{q}}_{\mathbf{i}}(t)+\frac{1}{e_{i}} \mathbf{B}_{\mathrm{i}}^{T} \xi_{\mathrm{i}}(t),
$$

where

$$
\mathbf{K}_{\mathbf{i}}(t)=\frac{1}{e_{i}} \mathbf{B}_{\mathrm{i}}^{T} \mathbf{S}_{\mathbf{i}}(t)
$$

It is apparent from Eq. (22) that the modal control input, $f_{i}(t)$, consists of two parts: the closed loop part, $-\mathbf{K}_{\mathbf{i}}(t) \overline{\mathbf{q}}_{\mathbf{i}}(t)$, which is dependent on the states and has a time varying feedback gain matrix $\mathbf{K}_{\mathrm{i}}(t)$; the open loop part, $\frac{1}{e_{\mathrm{i}}} \mathbf{B}_{\mathrm{i}}^{T} \xi_{\mathrm{i}}(t)$, which is independent of the current states. Figure 2 depicts the modal control block diagram used for the implementation of this tracking control problem. Both the dynamic model and the controller are shown. The regulator control design can be obtained by removing the open loop control part, namely, the disturbance due to the moving load is ignored. Note that the regulator formulation is optimal only when the disturbance due to the moving load is null. To synthesize the modal control force, the modal states, $\overline{\mathbf{q}}_{\mathrm{i}}(t)$, used as feedback, must be available to the control system. The modal states can be estimated using modal filters for actual implementation (Meirovitch and Baruh, 1982, 1985).

\section{NUMERICAL IMPLEMENTATION}

Numerical implementation considerations for the optimal control formulation are discussed in this section. The required computation for the Riccati matrix, the $\xi_{i}(t)$ vector for the open loop control, and the states of the dynamic model are addressed. Implementation consideration of the disturbance due to the moving load is also presented. For the optimal independent modal space control formulation presented here, a set of three nonlinear differential equations for elements in the Riccati matrix and another set of two linear differential equations with time-dependent coefficients for the $\xi_{\mathrm{i}}(t)$ vector have to be solved by integrating back in time for each mode controlled. Computational time is significantly reduced when compared with the coupled mode control scheme in which $2 n(2 n+1) / 2$ nonlinear Riccati equations and $2 n$ linear differential equations for the $\xi_{\mathrm{i}}(t)$ vector are to be solved.

There are different approaches to solve the nonlinear differential equations for the Riccati matrix (Anderson and Moore, 1971). In this work, the nonlinear equations are transformed to linear ones so that explicit expressions for $\mathbf{S}_{\mathbf{i}}(t)$ can be obtained without using the expensive step by step time integration scheme. The Riccati matrix can be solved as follows: 


$$
\mathbf{S}_{\mathrm{i}}(t)=\mathbf{Y}_{\mathbf{i}}(t) \mathbf{Z}_{\mathbf{i}}^{-1}(t),
$$

in which

$$
\left[\begin{array}{c}
\dot{\mathbf{Y}}_{\mathbf{i}} \\
\dot{\mathbf{Z}}_{\mathrm{i}}
\end{array}\right]=\mathbf{Q}_{\mathbf{i}}\left[\begin{array}{c}
\mathbf{Y}_{\mathbf{i}} \\
\mathbf{Z}_{\mathbf{i}}
\end{array}\right],
$$

with the terminal conditions

$$
\left[\begin{array}{c}
\mathbf{Y}_{\mathbf{i}}\left(t_{f}\right) \\
\mathbf{Z}_{\mathbf{i}}\left(t_{f}\right)
\end{array}\right]=\left[\begin{array}{c}
\mathbf{S}_{\mathbf{f}_{\mathbf{i}}} \\
\mathbf{I}
\end{array}\right]
$$

and

$$
\mathbf{Q}_{\mathbf{i}}=\left[\begin{array}{cc}
-\mathbf{A}_{\mathbf{i}}^{T} & -\mathbf{F}_{\mathbf{i}} \\
-\frac{1}{e_{i}} \mathbf{B}_{\mathbf{i}} \mathbf{B}_{\mathrm{i}}^{T} & \mathbf{A}_{\mathbf{i}}
\end{array}\right] .
$$

The state transition matrix in partitioned form for Eq. (24) is denoted as

$$
\boldsymbol{\Phi}_{\mathrm{i}}=\left[\begin{array}{ll}
\phi_{11_{\mathrm{i}}} & \phi_{12_{\mathrm{i}}} \\
\phi_{21_{\mathrm{i}}} & \phi_{22_{\mathrm{i}}}
\end{array}\right]
$$

with

$$
\mathbf{Q}_{\mathbf{i}}=\left[\begin{array}{cccc}
0 & \omega_{i} & -\omega_{i}^{2} & 0 \\
-\omega_{i} & 0 & 0 & -\omega_{i}^{2} \\
0 & 0 & 0 & \omega_{i} \\
0 & -\frac{1}{e_{i} \omega_{i}^{2}} & -\omega_{i} & 0
\end{array}\right]
$$

for the present problem, thus we have

$$
\begin{aligned}
& \mathbf{Y}_{\mathbf{i}}(t)=\phi_{11_{i}}\left(t-t_{f}\right) \mathbf{S}_{\mathbf{f}_{\mathbf{i}}}+\phi_{12_{i}}\left(t-t_{f}\right), \\
& \mathbf{Z}_{\mathbf{i}}(t)=\phi_{21_{i}}\left(t-t_{f}\right) \mathbf{S}_{\mathbf{f}_{\mathbf{i}}}+\phi_{22_{i}}\left(t-t_{f}\right),
\end{aligned}
$$

and from Eq. (23) we obtain

$$
\begin{aligned}
\mathbf{S}_{\mathbf{i}}(t)= & {\left[\phi_{1 \mathbf{1}_{\mathrm{i}}}\left(t-t_{f}\right) S_{\mathbf{f}_{\mathbf{i}}}+\phi_{1 \mathbf{2}_{\mathrm{i}}}\left(t-t_{f}\right)\right] } \\
& {\left[\phi_{2 \mathbf{1}_{\mathrm{i}}}\left(t-t_{f}\right) S_{\mathbf{f}_{\mathrm{i}}}+\phi_{22_{\mathrm{i}}}\left(t-t_{f}\right)\right]^{-1} . }
\end{aligned}
$$

Note that solution of the $\xi_{\mathrm{i}}(t)$ vector requires backtracking of the moving load as can be seen from Eq. (21) with the terminal condition being specified from the optimization process. Because the desired output, the $\eta_{\mathrm{i}}(t)$ vector, is zero, the only nonhomogeneous term is $\mathbf{S}_{\mathbf{i}}(t) \overline{\mathbf{P}}_{\mathrm{i}}(t)$ as shown in Eq. (21), which is integrated backward. Therefore, after the concentrated force leaves the beam span, the disturbance $\overline{\mathbf{P}}_{\mathrm{i}}(t)$ vector due to the moving force is zero and Eq. (21) becomes a set of homogeneous differential equations with terminal conditions being zero. This implies that the $\xi_{\mathrm{i}}(t)$ vector will be null for the time period when the moving load is outside the beam span. After the Riccati matrix, $\mathbf{S}_{\mathrm{i}}(t)$, and the $\xi_{\mathrm{i}}(t)$ vector are computed, they are used to synthesize the control input, $f_{i}(t)$, as shown in Eq. (19). The modal governing equation, Eq. (15), can then be integrated forward in time to obtain the modal response and the physical beam response can be obtained using Eq. (6).

\section{SIMULATION RESULTS}

It has been shown that when an elastic beam is subjected to a moving concentrated force, the dynamic response of the system is dominated by the fundamental mode with a very small contribution from the higher modes (Timoshenko, 1955). Therefore, control of the first mode with one actuator is considered here, which will be sufficient for practical vibration suppression of the present system. In this work, one actuator, located at the beam center, is used to control the vibration of the first mode so that minimum hardware for control implementation is required. It will be shown in this section that this configuration is sufficient to effectively suppress excessive vibration of the beam system. It should be noted that although the design in modal space is always optimal, disregard the choice of the actuator position as long as $\mathbf{B}^{-1}$ shown in Eq. (14) exists; the actuator position does affect the required amount of actual control input for control. Moreover, control spillover exists for a system controlled with discrete actuators as is evident in Eqs. (7)-(9). The choice of the beam center as the actuator position not only minimizes the required physical control input, but also reduces the spillover effect because the adjacent mode, the second mode in this analysis, and the higher antisymmetric modes are not excited due to actuation occurring at the nodal position. Control spillover to the symmetric modes, starting from the third mode, is present and this effect is accounted for in the numerical computation for this study. 


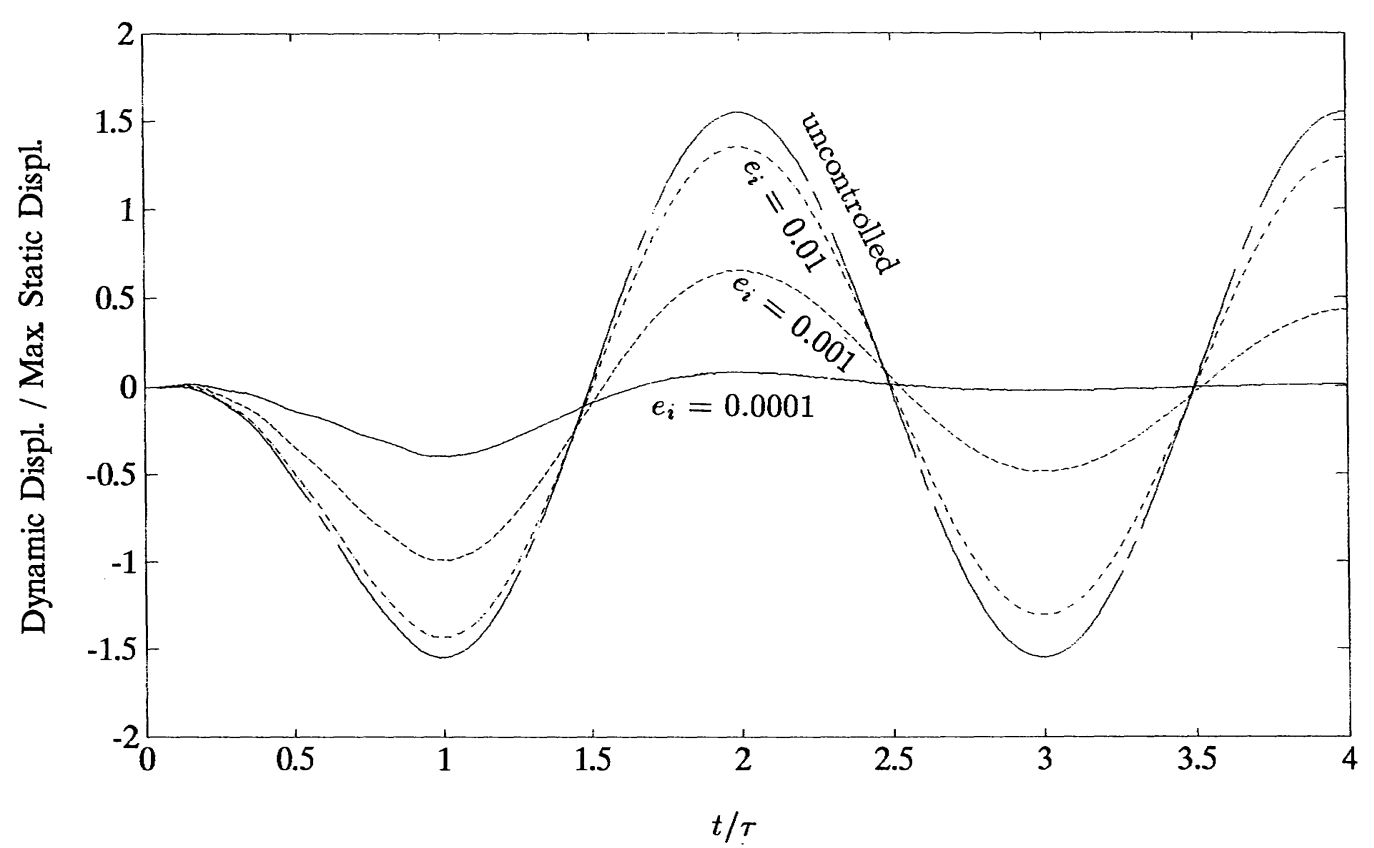

FIGURE 3 Dynamic displacement at the beam center with different weighting factors.

For the simulation study, the following set of numerical data was used: bending rigidity, $E I=$ $6.3487\left(10^{3}\right) \mathrm{Nm}^{2}\left(2.2122\left(10^{6}\right) \mathrm{lb} .-\right.$ in. $\left.^{2}\right)$, mass density per unit length, $\gamma=15.1 \mathrm{~kg} / \mathrm{m}\left(2.1901\left(10^{-3}\right)\right.$ lb.s ${ }^{2} /$ in. ${ }^{2}$ ), beam length, $L=1.1938 \mathrm{~m}$ (47 in.), and moving load magnitude, $F_{0}=444.8 \mathrm{~N}$ (100 lb.). The moving speed parameter of the concentrated force was $T_{\mathrm{f}} / \tau=2$, where $T_{\mathrm{f}}$ is the fundamental period of the support beam and $\tau$ is the time required for the moving force to travel from one end of the beam to the other. The final time, $t_{f}$, was chosen to be 4 times the fundamental period of the beam. The weighting matrix, $\mathbf{S}_{f_{i}}$, was chosen as zero for this study. Nine modes were used to compute the beam response.

The dynamic displacement and bending moment responses at the beam center and the physical active control input with different weights are shown in Figs. 3, 4, and 5, respectively. When the weighting factor for the control force is large, the beam response becomes greater because the control system tends to conserve the control energy due to its high cost. Note that the beam is virtually motionless after $t / \tau=2$, as is evident in the case represented by the solid curve shown in Figs. 3 and 4 for $e_{i}=0.0001$. The response at the beam center without active control is shown to serve as a reference for performance evaluation of the control system design. During the period of the beam being loaded by the moving force, the bend- ing moment histories are more irregular as shown in Fig. 4, which is due to slower convergence because bending moment involves the second derivative of the beam displacement. As can also be seen from Fig. 5, the control input is larger when a smaller value of weighting factor $e_{i}$ is used, which implies the control cost is less important. Table 1 summarizes the performance of both the optimal linear quadratic regulator (LQR) and the optimal linear quadratic tracking (LQT) control designs, in which $D_{y}$ and $D_{m}$ denote the dynamic impact factors defined as the maximum dynamic response normalized by the maximum static response for displacement and bending moment, respectively; $u_{\max }^{*}$ is the maximum amount of physical control input force normalized by the magnitude of the moving force; and the last row shows the performance index (PI) of the two control strategies with various weighting factors for the modal control force. The PI is defined as shown in Eq. (17). The percent deviation denotes the percent difference of the PI between the optimal LQT and LQR designs normalized by the PI of the optimal LQT approach. As can be seen in Table 1, the impact factors using the optimal LQR design are larger, with the maximum peak control input being smaller, when compared with those using the optimal LQT approach. The PI shown in the last row of Table 1 was used to assess the effectiveness of the two different control designs. The deviation 


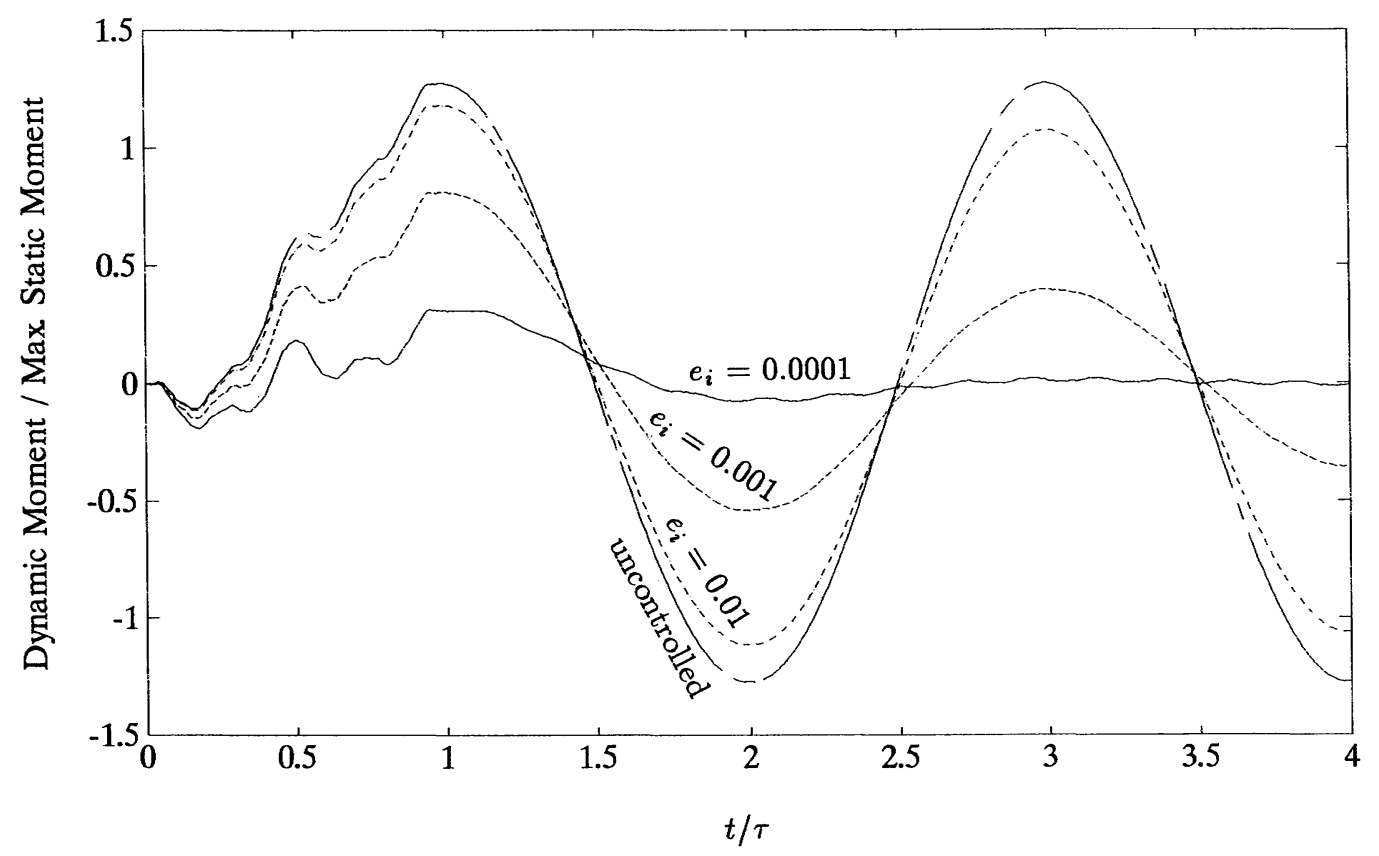

FIGURE 4 Dynamic bending moment at the beam center with different weighting factors.

of the optimal LQR design from the optimal LQT approach becomes larger as a smaller weighting factor for the control force is used. This indicates that the effectiveness of the optimal LQT approach becomes more appreciable than that of the optimal LQR design when more vibration sup- pression of the system is called for. The amount of peak control input to render the response performance, denoted by the solid line illustrated in Figs. 3 and 4, is 0.7417 times the magnitude of the moving force using the optimal LQT design. To further suppress the beam response, the required

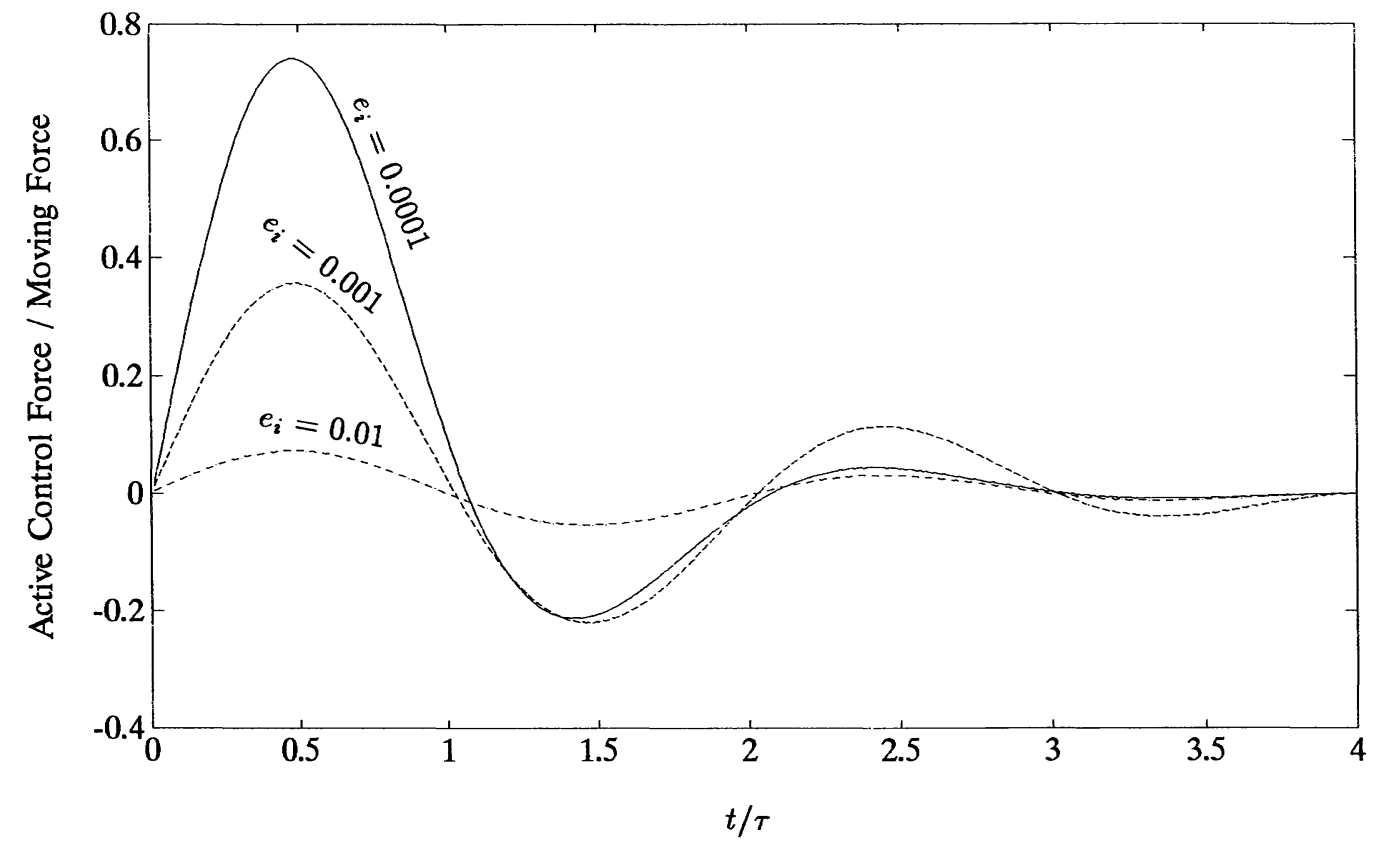

FIGURE 5 Active control input with different weighting factors. 
Table 1. Impact Factors (Displacement $D_{y}$, Moment $D_{m}$ ), Normalized Maximum Control Force $\left(u^{*}{ }_{\max }\right)$, and Performance Index (PI) for Different Weighting Factors $\left(e_{i}\right)$

\begin{tabular}{lccc}
\hline$e_{i}$ & 0.01 & 0.001 & 0.0001 \\
\hline$D_{y}$ & & & \\
LQT & 1.4346 & 0.9920 & 0.3954 \\
LQR & 1.4903 & 1.2382 & 0.7732 \\
$D_{m}$ & & & \\
LQT & 1.1806 & 0.8149 & 0.3194 \\
LQR & 1.2276 & 1.0221 & 0.6340 \\
$u_{\max }^{*}$ & & & \\
$\quad$ LQT & 0.0730 & 0.3574 & 0.7417 \\
LQR & 0.0547 & 0.2742 & 0.5681 \\
PI & & & \\
LQT & $8.3261\left(10^{4}\right)$ & $4.0726\left(10^{4}\right)$ & $8.4298\left(10^{3}\right)$ \\
LQR & $8.5145\left(10^{4}\right)$ & $4.5983\left(10^{4}\right)$ & $1.1634\left(10^{4}\right)$ \\
Deviation $(\%)$ & -2.2629 & -12.9082 & -38.0104 \\
\hline
\end{tabular}

control input will be increased for this control system. For reference, the impact factors $D_{y}$ and $D_{m}$ are 1.5478 and 1.2746 , respectively, for the uncontrolled beam system. Apparently, excessive vibration of the beam system subjected to a moving force can be effectively suppressed using the current control formulation with a minimum hardware requirement.
For some civil engineering structures subjected to moving loads, the magnitude and speed of the load may be known as a priori (Leipholz and Abdel-Rohman, 1986), and the optimal LQT approach can be used to yield the optimal vibration suppression performance. However, for some other mechanical structures, the characteristics of the moving load may not be accurately estimated.

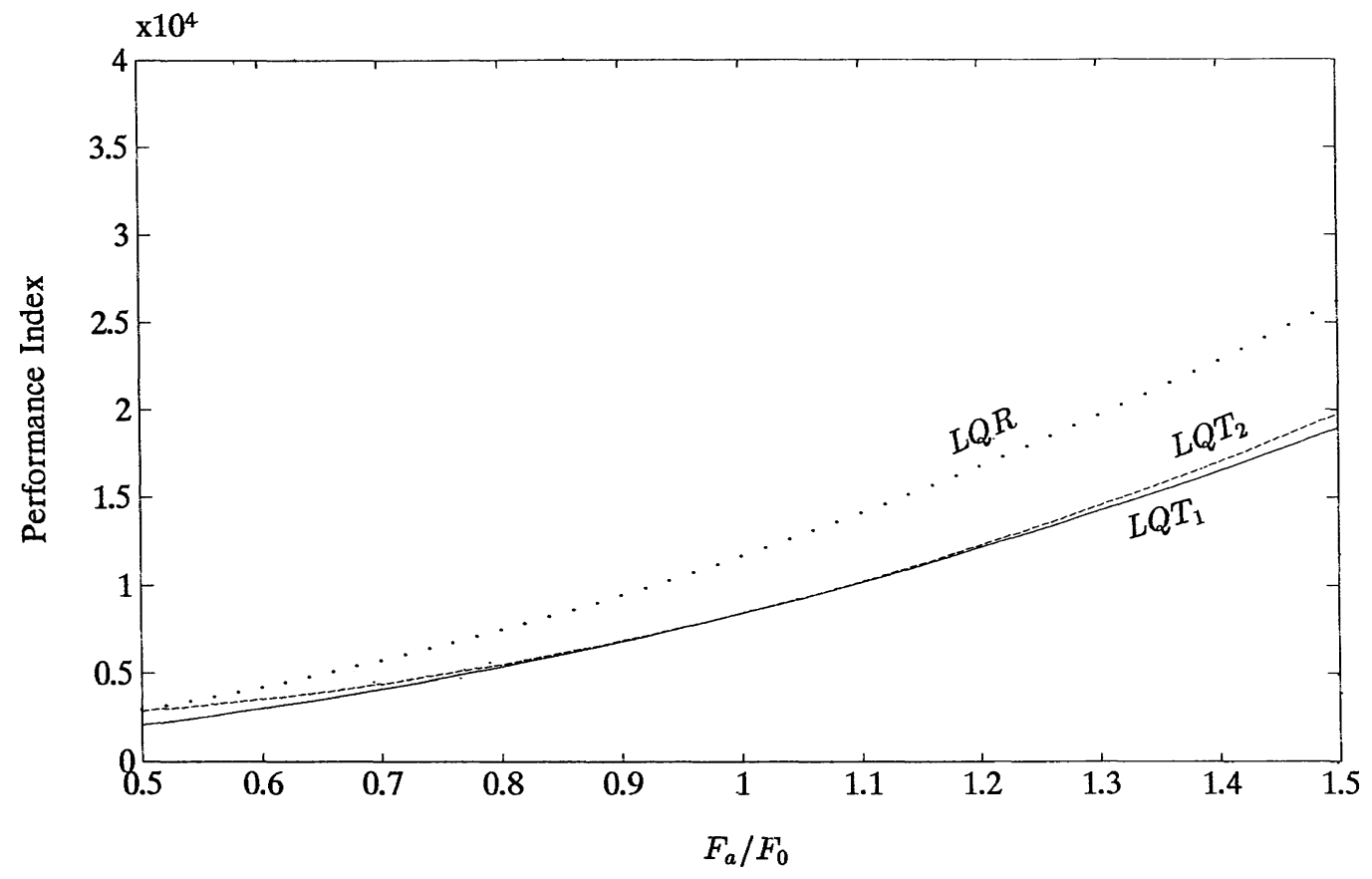

FIGURE 6 Effect of the moving load magnitude variation on performance index using optimal LQT and optimal LQR control designs. $\mathrm{LQT}_{1}$ : load magnitude known exactly; $\mathrm{LQT}_{2}$ : load magnitude known approximately with designed magnitude $F_{0}=444.8 \mathrm{~N}(100 \mathrm{lb}$.); LQR: disturbance due to moving load ignored. 


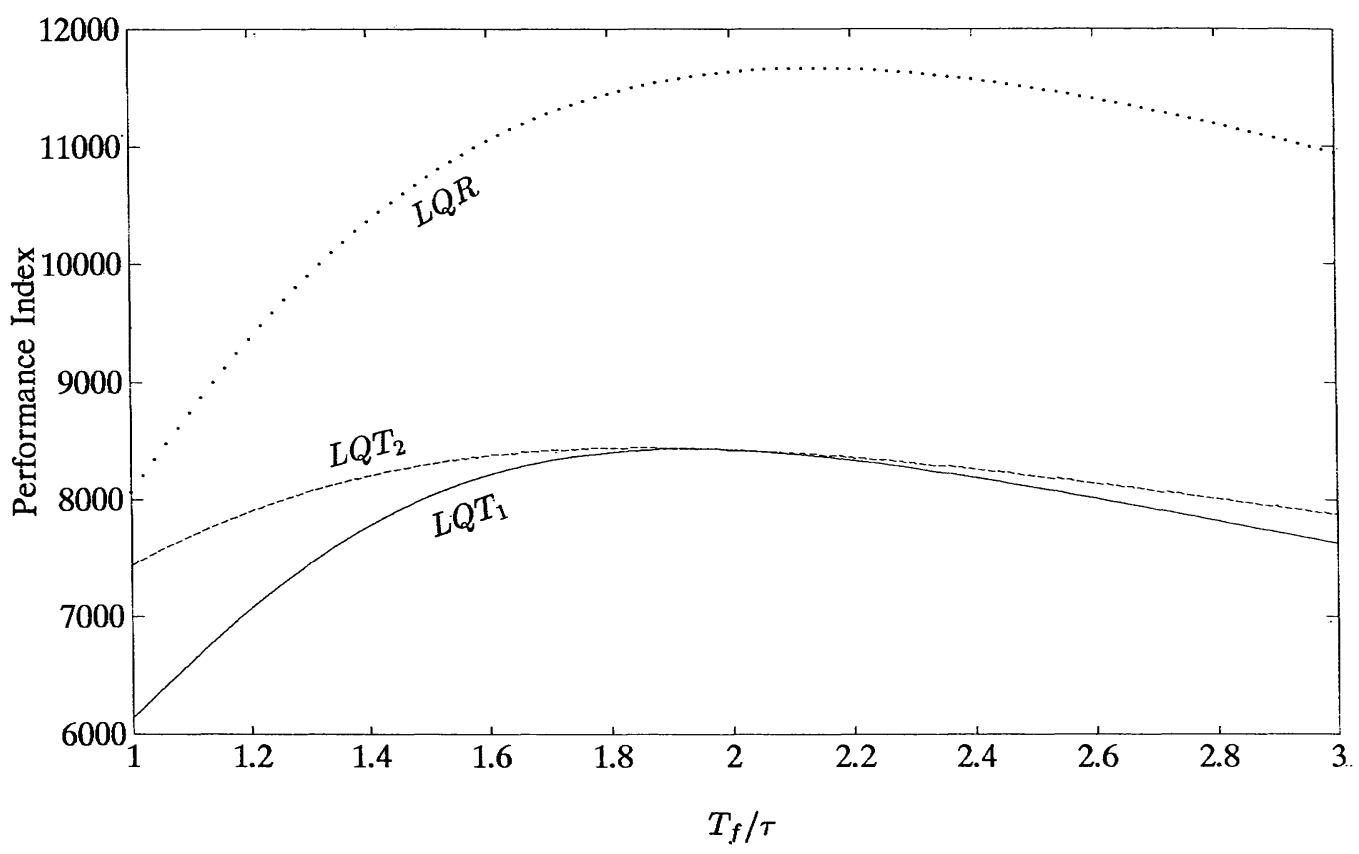

FIGURE 7 Effect of the moving load speed variation on performance index using optimal LQT and optimal LQR control designs. $\mathrm{LQT}_{1}$ : moving speed known exactly; $\mathrm{LQT}_{2}$ : moving speed known approximately with designed moving speed $T_{\mathrm{f}} / \tau=2$; LQR: disturbance due to moving load ignored.

Therefore, it is of interest to examine the performance of the tracking control approach as compared to that of the regulator control design when the actual magnitude and speed of the moving load deviate from that designed for the tracking control system. Note that the only difference between the tracking and the regulator control designs is that the former incorporates an additional open loop control by synthesizing the $\xi_{\mathrm{i}}(t)$ vector based on the knowledge of the moving load magnitude and speed. Figures 6 and 7 illustrate the performance comparison between the optimal LQT design and the optimal LQR approach with respect to the moving load magnitude and speed, respectively. The weighting factor for the modal control force is 0.0001 . For the analysis shown in Fig. 6, the design magnitude of the moving load was $F_{0}=444.8 \mathrm{~N}(100 \mathrm{lb}$.); the moving speed parameter, $T_{f} / \tau$, was 2 . The abscissa denotes the actual load magnitude, $F_{\mathrm{a}}$, normalized by the designed one, $F_{0}$. A range of \pm 50 variation from that designed in the moving load magnitude was analyzed. The solid line, denoted by $\mathrm{LQT}_{1}$, represents the true optimal tracking control performance where the magnitude of the moving load was known exactly. The dashed line, denoted by $\mathrm{LQT}_{2}$, shows the case where the tracking control system still used the designed moving load magnitude $F_{0}$ to synthesize the open loop control vector, without knowing the actual moving load magnitude. The dotted line represents the situation when the regulator control design was used. As can be seen in Fig. 6, the performance of the tracking control with inaccurate knowledge of the moving load magnitude only deviates slightly from the optimal tracking performance where the load magnitude is known exactly. It can be concluded from Fig. 6 that even with inaccurate information of the moving load magnitude, the tracking control approach always performs better than the regulator control design within the range of load variation considered here. In Fig. 7 the effect of speed variation of the moving load is examined. The designed moving load speed parameter, $T_{\mathrm{f}} / \tau$, was 2. The solid line, denoted by $\mathrm{LQT}_{1}$, describes the case where the moving load speed was known exactly, the dashed line, denoted by $\mathrm{LQT}_{2}$, represents the situation where the control system still used the designed moving load speed $T_{\mathrm{f}} / \tau=2$ to formulate the $\xi_{\mathrm{i}}(t)$ vector, without adapting to the true moving load speed. As can be seen in Fig. 7 , it can also be concluded that, within a $\pm 50 \%$ variation of moving load speed from that designed as shown in the present analysis, the tracking con- 
trol approach with inaccurate knowledge of the moving load speed still has better performance than the regulator control design. Note that inaccurate estimation of the moving load magnitude and speed can never destabilize the tracking control system because the $\xi_{\mathrm{i}}(t)$ vector is independent of the feedback modal states.

An alternative control formulation that does not require knowledge of the moving loads is the direct feedback control strategy. However, its noncollocated control version may lead to severe instability problems (Lin and Chu, 1995). Although the collocated design may provide improvement, the control is nonoptimal; that is, the optimal tracking control strategy as presented in this work provides an optimal method of structural vibration control due to moving loads when both the costs of system response and control input are taken into account and an objective function as depicted in Eq. (17) is considered. This consequence is logical because the optimal control system has complete knowledge of the moving load characteristics and hence can better react and take a more appropriate control action.

\section{CONCLUSIONS}

Vibration of structures subjected to moving loads can be significantly greater than that in the static case. For safety concerns, operation accuracy, or personnel comfort, appropriate means must be taken to mitigate the excessive structural vibration. In this work, optimal independent modal space control for vibration suppression of beam structures subjected to a moving concentrated force was presented. Excessive beam vibration can be effectively suppressed by using this control strategy with far less computational effort when compared to conventional coupled mode control techniques. The optimal tracking problem led to a control strategy including a combination of open loop and closed loop control and was found to be more effective than the optimal regulator control design, even with inaccurate estimation $( \pm 50 \%$ error) of the moving load magnitude or speed from that designed for the tracking control system. The required time varying feedback gains can be solved off-line, and hence real time control can be implemented without difficulties.

The beam vibration can be suppressed while keeping the amount of control input within a reasonable range. Minimum hardware with only a single actuator for the control of the fundamental mode was adopted, in consideration of the nature of the moving load effect. The performance of the control system depends on the choice of weighting matrices. In practice, the weighting matrices are chosen based on engineering considerations, i.e., the tolerable beam vibration, the magnitude limitation, and the cost of the control input. The designer is responsible for such choices. Future research topics include systems with closely spaced or repeated eigenvalues, time delay, nonlinearity, inelastic behavior, etc. Vibration control of support structures subjected to moving sprung dynamic systems, rather than moving concentrated forces, for more precise modeling, is also worth further exploration.

This author is grateful for the financial support of the National Science Council (NSC), ROC, under Grant NSC 81-0403-E-019-514.

\section{REFERENCES}

Anderson, B. D. O., and Moore, J. B., 1971, Linear Optimal Control, Prentice-Hall, Inc., Englewood Cliffs, NJ.

Baruh, H., and Silverberg, L., 1985, "Robust Natural Control of Distributed Systems," Journal of Guidance, Control, and Dynamics, Vol. 8, pp. 717-724.

Filho, F. V., 1978, "Finite Element Analysis of Structures under Moving Loads," Shock and Vibration Digest, Vol. 10, pp. 27-35.

Fryba, L., 1972, Vibration of Solids and Structures under Moving Loads, Nordhoff International Publishing, Groningen, The Netherlands.

Hino, J., Yoshimura, T., and Ananthanarayana, N., 1985, "Vibration Analysis of Nonlinear Beams Subjected to a Moving Load Using the Finite Element Method," Journal of Sound and Vibration, Vol. 100, pp. 477-491.

Hino, J., Yoshimura T., and Konishi, K., 1984, “'A Finite Element Method Prediction of the Vibration of a Bridge Subjected to a Moving Vehicle Load," Journal of Sound and Vibration, Vol. 96, pp. 45-53.

Katz, R., Lee, C. W., Ulsoy, A. G., and Scott, R. A., 1987, "Dynamic Stability and Response of a Beam Subjected to a Deflection Dependent Moving Load," Journal of Vibration, Acoustics, Stress, and Reliability in Design, Vol. 109, pp. 361-365.

Kirk, D. E., 1970, Optimal Control Theory, PrenticeHall, Inc., Englewood Cliffs, NJ.

Leipholz, H. H. E., and Abdel-Rohman, M., 1986, Control of Structures, Martinus Nijhoff Publishers, Boston.

Lin, Y.-H., and Cho, C.-H., 1993, "Vibration Suppression of Beam Structures Traversed by Multiple Mov- 
ing Loads Using A Damped Absorber," Journal of Marine Science and Technology, Vol. 1, pp. 39-48.

Lin, Y.-H., and Chu, C.-L., 1995, "Numerical Evaluation for Stability and Performance of an Electronic Damping Device for Structural Vibration Control,' Journal of Sound and Vibration, Vol. 184, pp. 929-933.

Lin, Y.-H., and Trethewey, M. W., 1990, "Finite Element Analysis of Elastic Beams Subjected to Moving Dynamic Loads," Journal of Sound and Vibration, Vol. 136, pp. 323-342.

Lin, Y.-H., and Trethewey, M. W., 1993, "Vibration Suppression of Beam Structures Subjected to Moving Loads: A Feasibility Study Using Finite Elements," Journal of Sound and Vibration, Vol.166, pp. 383-395.

Lin, Y.-H., Trethewey, M. W., Reed, H. M., Shawley, J. D., and Sager, S. J., 1990, "Dynamic Modeling and Analysis of a High Speed Precision Drilling Machine," Journal of Vibration and Acoustics, Vol. 112, pp. 355-365.

Meirovitch, L., and Baruh, H., 1982, "Control of SelfAdjoint Distributed-Parameter Systems," Journal of Guidance, Control, and Dynamics, Vol. 5, pp. 60-66.

Meirovitch, L., and Baruh, H., 1983, "Robustness of the
Independent Modal-Space Control Method," Journal of Guidance, Control, and Dynamics, Vol.6,pp.20-25. Meirovitch, L., and Baruh, H., 1985, "The Implementation of Modal Filters for Control of Structures," Journal of Guidance, Control, and Dynamics, Vol. 8, pp. 707-716.

Stokes, G. G., 1883, "Discussions of a Differential Equation Relating to the Breaking of Railway Bridges," Mathematical and Physical Papers, Vol. 2, pp. 178-220.

Su, Y.-A., and Tadjbakhsh, I. G., 1991, "Optimal Control of Beams with Dynamic Loading and Buckling," Transactions of the ASME Journal of Applied Mechanics, Vol. 58, pp. 197-202.

Timoshenko, S. P., 1955, Vibration Problems in Engineering, 3rd ed. D. Van Nostrand, New York.

Warburton, G. B., 1976, The Dynamic Behavior of Structures, Pergamon Press, New York.

Yoshimura, T., Hino, J., and Ananthanarayana, N., 1986, "Vibration Analysis of Nonlinear Beams Subjected to Moving Loads by Using the Galerkin Method," Journal of Sound and Vibration, Vol. 104, pp. 179-186. 

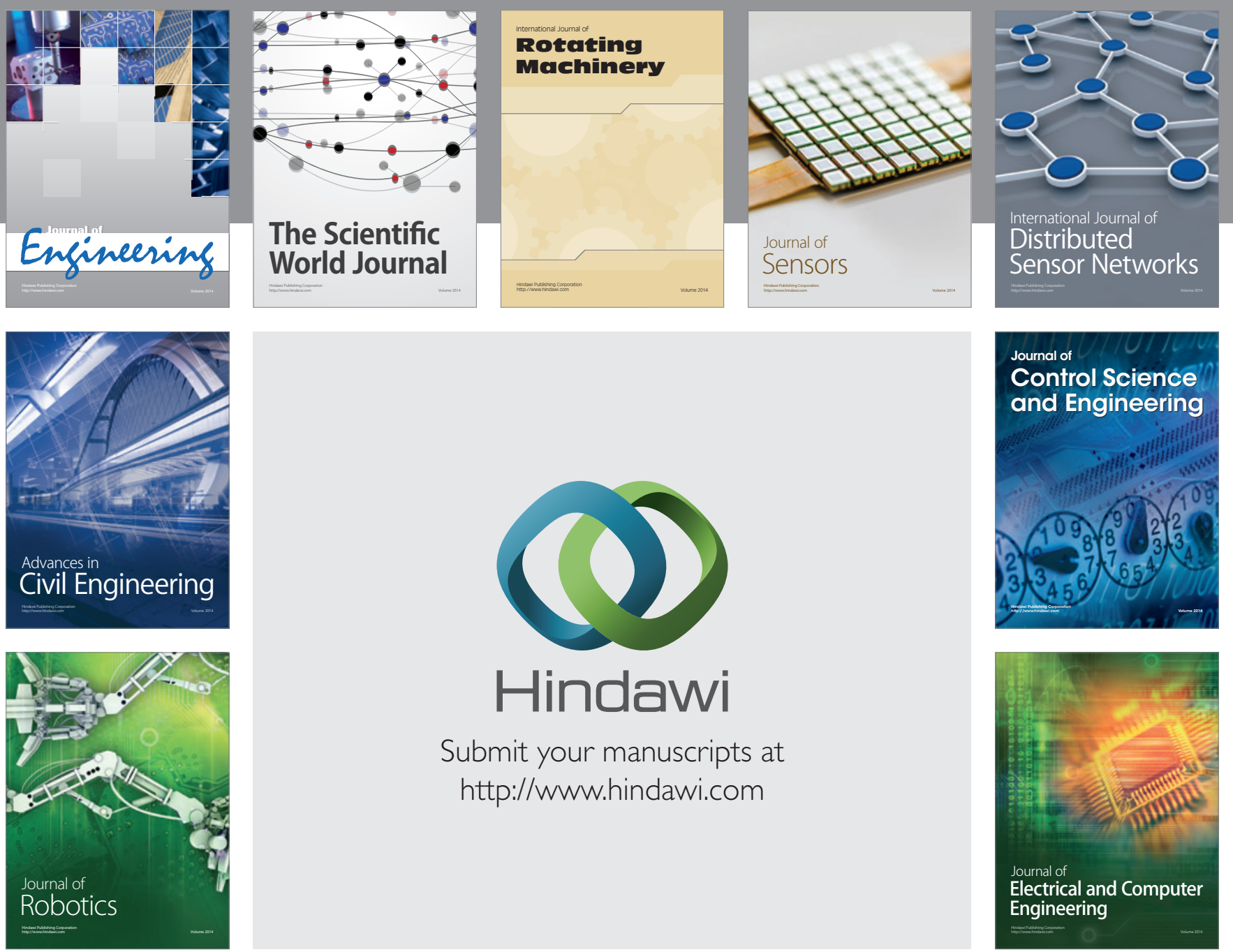

Submit your manuscripts at

http://www.hindawi.com
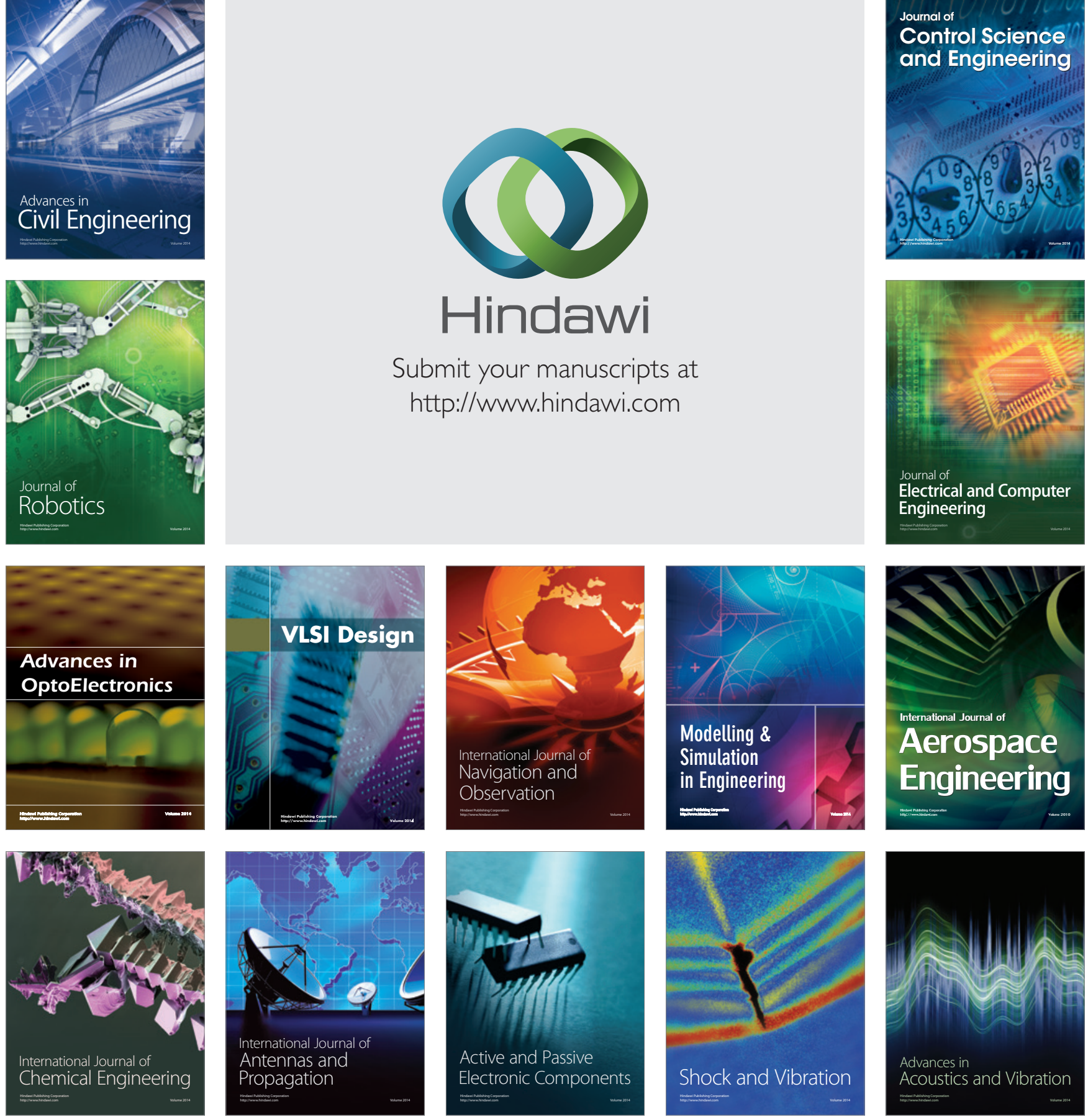